\title{
Useful Caregiving Characteristics and Interpersonal Cognitive Complexity
}

\author{
N. Swink and L. Medvene \\ Department of Community Psychology, College of Liberal Arts and Sciences
}

\section{Introduction}

Currently long-term care facilities for the elderly are aligning themselves behind a cause which has come to be known as a "culture change". This culture change has, as one of several established goals, the objective of making long-term care facilities more like homes, and less like institutions. Our research was aimed at identifying a relationship between three characteristics thought to be of value in more home-like care facilities, and the Interpersonal Cognitive Complexity (ICC) of nursing students exhibiting such characteristics. Three characteristics important in home-like caregiving situations were: (1) more extensive person perception, (2) less extreme peer judgments, and (3) more balanced descriptions of others. ICC is a way of looking at, or talking about, the number of internal constructs one has available to process information associated with the description and perception of others.

A linear relationship was hypothesized between ICC and each of the three characteristics. Specifically, we expected: (1) that those higher in ICC would demonstrate more perception of detail on a video-based person-perception task; (2) that as ICC of participants increased the characteristics listed to describe others would be less extreme; and (3) that participants with higher ICC would demonstrate having more balanced (in terms of the number of characteristics listed) descriptions of peers, regardless of whether the participant liked or disliked the peer being described.

\section{Experiment}

\section{Participants}

Female ( $n=33$ ) and male ( $n=7)$ nursing students from one first-year nursing class participated in the study for course credit. Ten class members chose not to participate. Participants were between 20 and 51 (M= 29.5, SD= 8.6) years old and 95\% had completed at least their sophomore year in college. The population was largely Caucasian (80\%), with relatively few participants identifying themselves as Asian (7.5\%), Hispanic (5\%) African American (5\%), and “Other” (2.5\%).

\section{Materials}

$R C Q$. ICC was operationalized within the study as score on the abridged version of the Role Category Questionnaire [1] which has been shown to be as effective and less obtrusive compared to it's predecessor [2]. The version used here consists basically of asking participants to generate names of two peers they know, one the participant likes, and one that the participant doesn't like, and then listing characteristics to describe each. It is thought that the number of characteristics a person lists is in some way reflective of the number of constructs that person has available to describe and characterize others.

Entry unitizing. In order to ensured a uniform interpretation of all entries, all participants' completed RCQ forms were typed (exactly as written) by a research assistant. This work was supervised by a researcher.

Codebooks. Crockett's [2] codebook for the RCQ served as the basis for our own RCQ codebook which governed all scoring decisions. Our RCQ codebook developed as the coding unfolded. In the end, the codebook consisted of a set of rules for counting a characteristic as construct (score of 1) or not (a score of 0 ) with each rule followed by a list of clarifying examples complete with explanations. A second codebook was created to outline the rules for counting the listed constructs as either "positive" or "negative” as this data was needed for the extremity of judgment hypothesis.

Coders. Four female Wichita State University undergraduate Psychology students coded responses to the RCQ for course credit. Inter-rater reliability among coders ranged from .95-.97 for all coding tasks.

Videotaped biography. A 15-minute videotaped biography of a man named Carl was created in collaboration with the Cramer Reed Center for Successful Aging, Larksfield Place. The video is a condensed version of a 45 minute interview with Carl featuring brief stories about his childhood, career, marriage, hobbies, and retirement years.

\section{Procedures}

Time I. Nursing students were administered the RCQ in their regular classroom at the beginning of class. Over the course of the next two weeks, coders scored the entries in accordance with the RCQ codebook, ultimately categorizing participants as 
either high (above median) or low (below median) in ICC. Two groups of equal size were created, each containing an equal number of participants high and low in ICC.

Time II. Two weeks after our initial visit, researchers returned to the nursing students' classroom and announced the two groups. Students participating in the research were led into a nearby classroom where they watched the videotaped biography of Carl along with the group to which they had been was assigned and one researcher. Following the video presentation, participants were asked to describe Carl on a form modeled after the RCQ form.

\section{Results}

Person Perception. Here we predicted that a positive correlation would exist between participants' RCQ scores and the mean number of constructs participants used to describe Carl following presentation of the video biography. A significant positive correlation was found between participants' RCQ scores and the mean number of constructs used to describe Carl $r$ $(37)=.58, p<.01$.

Extremity of Peer Judgments. In this case we predicted that the group mean of the variable representing extremity of judgment would correlate positively with participants' mean RCQ score. A non-significant positive correlation was found between RCQ score and the extremity of judgment variable $r(40)=.28, p=.84$.

Balance of Peer Descriptions. In addressing the third hypothesis, we predicted that a variable created to represent the ratio of constructs listed for liked vs. disliked peers would correlate positively with participants' RCQ scores. A significant positive correlation was found between these two variables $r(40)=.32, p<.05$.

\section{Discussion}

Two findings of the current research were of particular interest. First, there was a strong association between one's interpersonal cognitive complexity and his or her complexity of performance on a person perception task (i.e. how thoroughly he or she described Carl, the subject of the video biography). Secondly, there was a linear relationship between interpersonal cognitive complexity and the ratio of characteristics listed for a liked, and a disliked, peer. To clarify, the higher a person's overall interpersonal cognitive complexity, the more likely it was that the person used as many constructs to describe a disliked peer as to describe a liked peer. In contrast, a person with very low interpersonal cognitive complexity was more likely to list a greater number of descriptors for a liked, as opposed to a disliked peer. This expands our current understanding that generally everyone describes those we like in more detail than those we don't like [1].

\section{Conclusions}

These results lend support to a growing body of literature suggesting that caregivers with more ICC are likely to also display characteristics which are more desirable for caregivers. This suggests that the RCQ might find utility as a screening tool, and as an outcome or evaluation measure for nursing homes undergoing the current culture change.

\section{Acknowledgements}

The authors would like to thank Kate Harkness, not only for allowing us the class time and opportunity to enlist her students as participants, but also for her thoughtful insights which have proved helpful to us along the way.

\section{References}

[1] Burleson, B.R., \& Caplan, S.E. (1998). Cognitive complexity. In J.C. McCroskey, J.A. Daly, M.M. Martin \& M.J. Beatty (Eds.), Communication and personality: Trait perspectives. Cresskill, NJ: Hampton Press.

[2] Crockett, W.H. (1965). Cognitive complexity and impression formation. In B.A. Maher (Ed.) Progress in experimental personality research, vol. 2 (pp. 47-90). New York: Academic Press. 